\title{
Alpha-2, 3-sialyltransferases regulate the multidrug resistance of chronic myeloid leukemia through miR-4701-5p targeting ST3GAL1
}

\author{
Yan Li $i^{1,2,7}$, Shihua Luo ${ }^{3,7}$, Weijie Dong ${ }^{4}$, Xiaobo Song ${ }^{5}$, Huimin Zhou ${ }^{6}$, Lifen Zhao ${ }^{1}$ and Li Jia ${ }^{1}$
}

The aberrant sialylation profile on the surface of leukemia cells has been recognized for its potential diagnostic value towards assessing leukemia multidrug resistance (MDR). MicroRNAs as endogenous regulators of gene expression have been implicated in treating MDR. In this study, we describe the differential expressional profiles of $a$-2, 3-sialyltransferases (ST) and miR-4701-5p in three pairs of chronic myeloid leukemia (CML) cell lines and 48 clinical samples of bone marrow mononuclear cells from CML patients. The altered expression level of ST3GAL1 was found corresponding to the drugresistant phenotype (with and without adriamycin resistance) of CML cell lines both in vitro and in vivo. Further the results showed that miR-4701-5p directly targeted ST3GAL1 to reduce CML cells resistance to multiple chemotherapeutics in vitro and to convert tumor cells from adriamycin resistant to susceptible in vivo of mice. These results indicate that differential expression of $a-2,3$ ST is involved in MDR of CML, and that miR-4701-5p regulates the susceptibility of CML cells to multiple drugs, at least in part, through targeting ST3GAL1.

Laboratory Investigation (2016) 96, 731-740; doi:10.1038/labinvest.2016.50; published online 18 April 2016

Chronic myeloid leukemia (CML) is a clonal hematopoietic stem cell disorder characterized by extensive proliferation and expansion of granulocytic cells of varying stages of maturation and differentiation. ${ }^{1}$ Multidrug resistance (MDR) of leukemia cells is a defense mechanism for retaining homeostasis when they are damaged by cytotoxic drugs. Classic MDR is the consequence of overexpression of transporter proteins that belong to the ATP binding cassette family such as Pglycoprotein. ${ }^{2}$ In recent years, much research has been conducted on understanding the relationship between glycan alterations and resistance to chemotherapy of malignant cells. The changes of glycans attached on cell glycoproteins have been linked to drug resistance in leukemia. ${ }^{3,4}$ Therefore, much attention has been paid on regulation of glycosyltransferases in an effort to overcome MDR in the treatment of CML.

Human sialyltransferases (STs) are a group of enzymes responsible for transferring of sialic acid from cytidine 5-prime monophospho-N-acetylneuraminic acid (CMPNeuAc) to terminal positions of glycoprotein and glycolipid carbohydrate groups. ${ }^{5}$ Up to 20 different human STs have been characterized and classified according to the specific sialic acid linkage. Alpha-2, 3-STs mediate the sialic acid with an $\alpha$-2, 3-linkage to the terminal Gal residues (ST3Gal I-VI). ${ }^{5}$ Changes in the expression level of specific $\alpha$-2, 3-STs have been observed in several tumors. ST3Gal I or II was upregulated in human bladder cancer tissues or renal carcinoma cells. ${ }^{6,7}$ The most over-expressed ST in breast tumor was ST3Gal III, and its expression was positively correlated with tumor size and the number of axillary nodes involved. ${ }^{8}$ High level of ST3Gal IV expression was positively related to the malignant behavior of gastric cancer cells. ${ }^{9}$ ST3Gal V mRNA was up-regulated in lymphoblasts of acute lymphoblastic leukemia and closely associated with the high risk of the disease. ${ }^{10}$ ST3Gal VI was also up-regulated in melanoma cells. ${ }^{11}$

MicroRNAs (miRNAs) are a class of noncoding RNAs that are 21-25 nucleotides in length that function as the regulators of gene expression by targeting mRNAs for cleavage or translational repression. ${ }^{12}$ Tremendous evidence has shown

'College of Laboratory Medicine, Dalian Medical University, Dalian, Liaoning, China; ${ }^{2}$ Department of Clinical Laboratory, The First Affiliated Hospital of Dalian Medical University, Dalian, Liaoning, China; ${ }^{3}$ Department of Traumatology, Shanghai Ruijin Hospital, Jiaotong University, Shanghai, China; ${ }^{4}$ Department of Biochemistry, Dalian Medical University, Dalian, Liaoning, China; ${ }^{5}$ Department of Medical Biology, Faculty of Health Sciences, University of Troms $\varnothing$, Troms $\varnothing$, Norway and ${ }^{6}$ Department of Microbiology, Dalian Medical University, Dalian, Liaoning, China

Correspondence: Professor L Jia, PhD, College of Laboratory Medicine, Dalian Medical University, 9 Lvshunnan Road Xiduan, Dalian, Liaoning 116044, China.

E-mail: jiali0081@sina.com

7 These authors contributed equally to this work.

Received 1 November 2015; revised 1 March 2016; accepted 5 March 2016 
that various types of miRNAs participate in the regulation of many biological processes, ${ }^{13,14}$ affect cancer prognosis ${ }^{15}$ and influence the host response to chemotherapy. ${ }^{16,17}$ MiR-181b was decreased significantly in human multidrug-resistant leukemia cells and increased the sensitivity of leukemia cells to cytotoxic chemotherapeutic agents. ${ }^{18}$ MiR-381 and miR-495 modulated the MDR phenotype of leukemia K562/ $\mathrm{ADM}$ cells and were associated inversely with the expression of the MDR1 gene and its protein product, $\mathrm{P}$-glycoprotein. ${ }^{19}$ Altered expression of miR-331-5p and miR-27a were correlated with leukemia cell resistance and relapse. ${ }^{20}$ MiR-138 also reversed MDR of leukemia HL-60/VCR cells and promoted adriamycin-induced cell apoptosis. ${ }^{21}$ However, the role of miR-4701-5p in anticancer drug resistance by targeting ST3GAL1 remained unknown.

In the present study, we investigated the expression levels of $\alpha-2,3$-STs, and miR-4701-5p in parental and chemoresistant CML cell lines and in the bone marrow mononuclear cells (BMMC) isolated from the diagnosed CML and CML/ adriamycin-resistant (ADR) patients, in order to identify the functional role of ST3GAL1 in cancer drug resistance in vitro and in vivo. Moreover, we elucidated the regulatory pathway involving miR-4701-5p and ST3GAL1 in CML cell MDR development.

\section{MATERIALS AND METHODS \\ Parental CML Cell Culture}

Three CML cell lines, KCL22, K562, and KU812 were obtained from the KeyGEN Company (Nanjing, China). All cell lines were cultured in RPMI 1640 medium (Gibco, Grand Island, NY, USA) supplemented with $10 \%$ heat inactivated fetal bovine serum (Sigma, St. Louis, MO, USA) and 1\% penicillin-streptomycin (Gibco, Grand Island, NY, USA) at $37^{\circ} \mathrm{C}$ in a humidified atmosphere containing $5 \% \mathrm{CO}_{2}$. Adriamycin (Sigma, St. Louis, MO, USA) was added to parental cell cultures in stepwise increasing concentrations from 1 to $5 \mathrm{mg} / \mathrm{l}$ for 6 months to develop an ADR subline, namely KCL22/ADR, K562/ADR, and KU812/ADR, respectively. To maintain the MDR phenotype, the complete medium of the resistant cell clones were supplemented with $1.0 \mathrm{mg} / \mathrm{l}$ adriamycin. Over $90 \%$ of ADR cells were susceptible to subsequent treatments if they were maintained in complete medium without adriamycin for 1 week.

\section{Samples of Patients}

Overall 48 untreated CML patients comprising 29 males and 19 females with age ranging from 17 to 70 years (median age of 43 years) and 6 healthy donors were recruited from the First Affiliated Hospital of Dalian Medical University (Dalian, China) from July 2011 to June 2014. They all provided written informed consent. Both the study and the contents of the written consent were approved by the institutional ethics committees. The diagnosis of CML was based on cytomorphology, cytochemistry, multiparameter flow cytometry, immunology, molecular genetics, and cytogenetics. Bone marrow samples were collected at diagnosis and before treatment. BMMC were isolated by density gradient separation using Lympholyte- $\mathrm{H}$, immediately mixed with TRIzol $(1 \mathrm{ml})$ and frozen at $-80^{\circ} \mathrm{C}$ until RNA were extracted.

\section{Real-Time PCR Analysis}

Total RNA was isolated from the BMMC samples and CML cell lines with the RNeasy Mini Kit (QIAGEN, Valencia, CA, USA), and cDNA was synthesized using QuantiTect Reverse Transcription Kit (QIAGEN, Valencia, CA, USA) according to the manufacturer's protocol. The expression of miR-4701$5 p$ was determined by using mirVanaTM quantitative realtime PCR (qRT-PCR) miRNA Detection Kit according to the manufacturer's protocol (Ambion Inc., Austin, TX, USA) and was normalized with the $2^{-\Delta \mathrm{CT}}$ method relative to U6-small nuclear RNA.

ST3GAL mRNAs were quantified by SYBR-Green-qRTPCR (Takara, Otsu, Shiga, Japan) and normalized to GAPDH. The sequences of the upstream and downstream primers were as follows: $5^{\prime}$-CAGAGATGGACGGTCACT- $3^{\prime}$ and 5'-CAACT GTGGTTTCTGACG-3' for ST3GAL1; 5'-GTGCCTCCGAC TGG TTTG-3' and $5^{\prime}$-GAAGCGGTAGGGGTTCT C-3' for ST3GAL2; 5'-TATGCTTCA GCCTTGATG-3' and 5'-TTGG TGACTGACAAGATGG-3' for ST3GAL3; 5'-ATG TTGGC TCTGGTCCTG-3' and 5'-AGGAAG ATGGGCTGATCC-3' for ST3GAL4; 5'-CAAAGCAAGATGAGAAGG-3' and $5^{\prime}$-AA ACTTGGGACGACAT TC $-3^{\prime}$ for ST3GAL5; $5^{\prime}$-ATGTCTAT TGGGTGGCAC- $3^{\prime}$ and $5^{\prime}$-CGCACACAGAAAAGGGT G-3' for ST3GAL6; 5'-CTCCTCCACCTTTGACGCTG-3' and 5'-T CCTCTTGTGC TCTTGCTGG-3' for GAPDH. The expression level of the target genes was determined relatively to GAPDH and calculated as $2^{-\Delta C T}$. All reactions were performed in triplicate.

\section{Western Blot Analysis}

Whole cell proteins were electrophoresed under reducing conditions in $10 \%$ polyacrylamide gels. The separated proteins were transferred to a polyvinylidene difluoride membrane. After blocking with 5\% skimmed milk in PBS containing $0.1 \%$ Tween 20 (PBST), the membrane was followed by overnight incubation at room temperature with ST3Gal I, ST3GalII, ST3Gal III, ST3GalIV, ST3Gal V, ST3Gal VI antibody (1/1000 diluted; Abcam, ab96129, ab96028, ab135853, ab87114, ab155671, ab103643, Cambridge, UK). The membrane was washed three times with PBS containing $0.1 \%$ Tween at $\mathrm{pH} 7.4$, incubated with horseradish peroxidase-conjugated anti-rabbit IgG (1/10 000 diluted; GE Healthcare UK Ltd., Little Chalf ont, UK) for $1 \mathrm{~h}$ at room temperature. A GAPDH antibody (1/200 diluted; Santa Cruz Biotech) was used as a control. All bands were detected using ECL Western blot kit (Amersham Biosciences, UK), according to the manufacturer's instruction. The bands were analyzed with LabWorks (TM ver4.6, UVP, BioImaging Systems). 


\section{Regulation of ST3GAL1 Gene Expression}

The coding sequence of wild-type ST3GAL1 was obtained and inserted into the NotI and BamHI sites of the pGLV5/H1/GFP +Puro lentiviral plasmid. The ST3GAL1 shRNA sequence was inserted into the BamHI and EcoRI sites of the pGLV3/H1/ GFP+Puro lentiviral plasmid. Lentivirus plasmids were cotransfected with PG-P1-VSVG, PG-P2-REV, and PG-P3-RRE plasmids into $293 \mathrm{~T}$ cells (Invitrogen), and virus-containing supernatants were prepared according to manufacturer's instructions. For lentivirus infection, cells were cultured in six-well-tissue culture plates and infected the lentiviral vectors at a multiplicity of infection of 40 for $24 \mathrm{~h}$. The medium was replaced with fresh complete medium. After 2 days, cells were observed by fluorescence microscopy to confirm that greater than $90 \%$ of the cells were GFP positive. Subsequently, the GFP-positive cells were screened by addition of $5 \mu \mathrm{g} / \mathrm{ml}$ puromycin. Then cells were collected for gene expression assay and for further explorations. The sequences of ST3GAL1 shRNA were listed as follows: shRNA1, 5'-ATTG ATGGAGGCCAAGGTG-3'; shRNA2, 5'-TGAACCTC TCAT CGAACCA-3'; shRNA3, 5'-AGACTCAAAGTCTGCATCG-3'. The sequence of scrambled shRNA was 5'-TTCTCCGAA CGTGTCACGT-3'.

\section{Flow Cytometry Analysis}

Cells were collected and blocked in 2\% BSA in PBS for $30 \mathrm{~min}$ and washed two times with PBS. After incubation of cells with fluorescein isothiocyanate (FITC)-MAL/LCA at a final concentration $(10 \mu \mathrm{g} / \mathrm{ml})$ in the dark for $40 \mathrm{~min}$ at room temperature, residual unbound FITC-MAL/LCA was then discarded by repeat centrifugation of samples at $1000 \mathrm{~g}$. The control, which was negative, cells and FITC-MAL/LCA was alone. Cells were collected and analyzed with Flow Cytometer (BD FACSAria, San Diego, CA, USA). Experiments were repeated at least three times with similar results each time.

\section{Oligonucleotide Construction and Luciferase Activity Assay}

Mimics, agomir, antagomir, and negative control oligonucleotides for hsa-miR-4701-5p were obtained from RiboBio Co. Ltd (GenePharma, Shanghai, China). The pGL3-MC firefly luciferase plasmids containing wild-type ST3GAL1 and mutant ST3GAL1 were synthesized (Promega). The sequences used were $5^{\prime}$-UUGGCCACCACACCUACCCCUU- $3^{\prime}$ and 5'-GGGGUAGGUGUGGUGGCCA AUU-3' (miR-4701-5p mimics), 5'-UUCUCCGAACGUGUCACGUTT- $3^{\prime}$ and $5^{\prime}$-A CGUGACACGUUCGGAGAATT-3' (mimics control), 5'-AA GGGGUAGGUGUG GUGGCCAA- $3^{\prime}$ (miR-4701-5p antagomir) and 5'-CAGUACUUUUGUGUAGUAC AA-3' (antagomir control).

The target cells were co-transfected with $300 \mathrm{ng}$ of the pGL3-MC firefly luciferase plasmids with the wild-type or mutated 3'-UTR sequences of ST3GAL1 and $60 \mathrm{ng}$ of pRL-TK Renilla luciferase plasmid (Promega), together with $30 \mathrm{nM}$ miR-4701-5p mimics or miR-control using Lipofectamine
2000 (Invitrogen). The lysates were collected $48 \mathrm{~h}$ after the transfection, and the activities of firefly and Renilla luciferases were measured by using the Dual-Luciferase reporter system (Promega) and normalized to those of Renilla luciferase activities. The mean of the results from the cells transfected with the miR-control was set at 1 . All experiments were performed in triplicate. The results were statistically analyzed using Student's $t$-test.

\section{In vitro Drug Susceptibility Assay}

Drug susceptibility was measured with MTT assay. The cells $\left(1 \times 10^{4}\right)$ were plated in 96-well plate and incubated with different anticancer drugs adriamycin, paclitaxel, and vincristine (Sigma, St. Louis, MO, USA) for $48 \mathrm{~h}$, respectively. Then cells were treated with $100 \mu \mathrm{l}$ MTT $(5 \mathrm{mg} / \mathrm{ml}$, Sigma, St. Louis, MO, USA). After $4 \mathrm{~h}$ incubation at $37^{\circ} \mathrm{C}$ in $5 \% \mathrm{CO}_{2}$, $100 \mu \mathrm{l}$ dimethylsulfoxide (DMSO, Gibco) was pipetted to solubilize the form azan product for $30 \mathrm{~min}$ at room temperature. The spectrometric absorbance was measured at $490 \mathrm{~nm}$ by microplate reader (Model 680; Bio-Rad, Hercules, CA, USA). Each group contained three wells and was repeated three times. The drug resistance was estimated by comparing the $\mathrm{IC}_{50}$ values (the drug concentration that inhibits cell growth by $50 \%$ ) from growth inhibition curves.

\section{In vivo Antitumor Activity}

Overall 5-week-old male athymic nude mice were obtained from the Animal Facility of Dalian Medical University, and were fed with sterilized food and water. Approximately $1 \times 10^{7}$ cells were injected subcutaneously into the right flank of each nude mouse, respectively. When the mice bearing palpable tumors (about 1 week after tumor cell inoculation), they were randomly divided into control and treatment groups (24 animals per group). The treatment groups received $7 \mathrm{mg} / \mathrm{kg}$ adriamycin i.p. three times a week for 3 weeks, and the control groups received physiological saline alone. The mice were humanely killed and their tumors were isolated, weighed, and photographed. The tumor volume was calculated by the following formula: tumor volume $=1 / 2\left(\right.$ length $\times$ width $\left.^{2}\right)$. These experiments were approved by the Committee on the Ethics of Animal Experiments of the Dalian Medical University, China.

\section{Statistical Analysis}

The data were expressed as the mean \pm standard deviation (s.d.) and analyzed by SPSS 13.0 software. Student's $t$-test was selected to determine the significance of differences among the examined groups. $P<0.05$ was considered to be statistically significant.

\section{RESULTS}

Differential Expression of ST3GAL Family in Three Pairs of Parental and Chemoresistant CML Cell Lines and CML Patients

It has been well established that the extent of $\mathrm{N}$-glycan sialylation depends on STs. Here, we performed real-time 

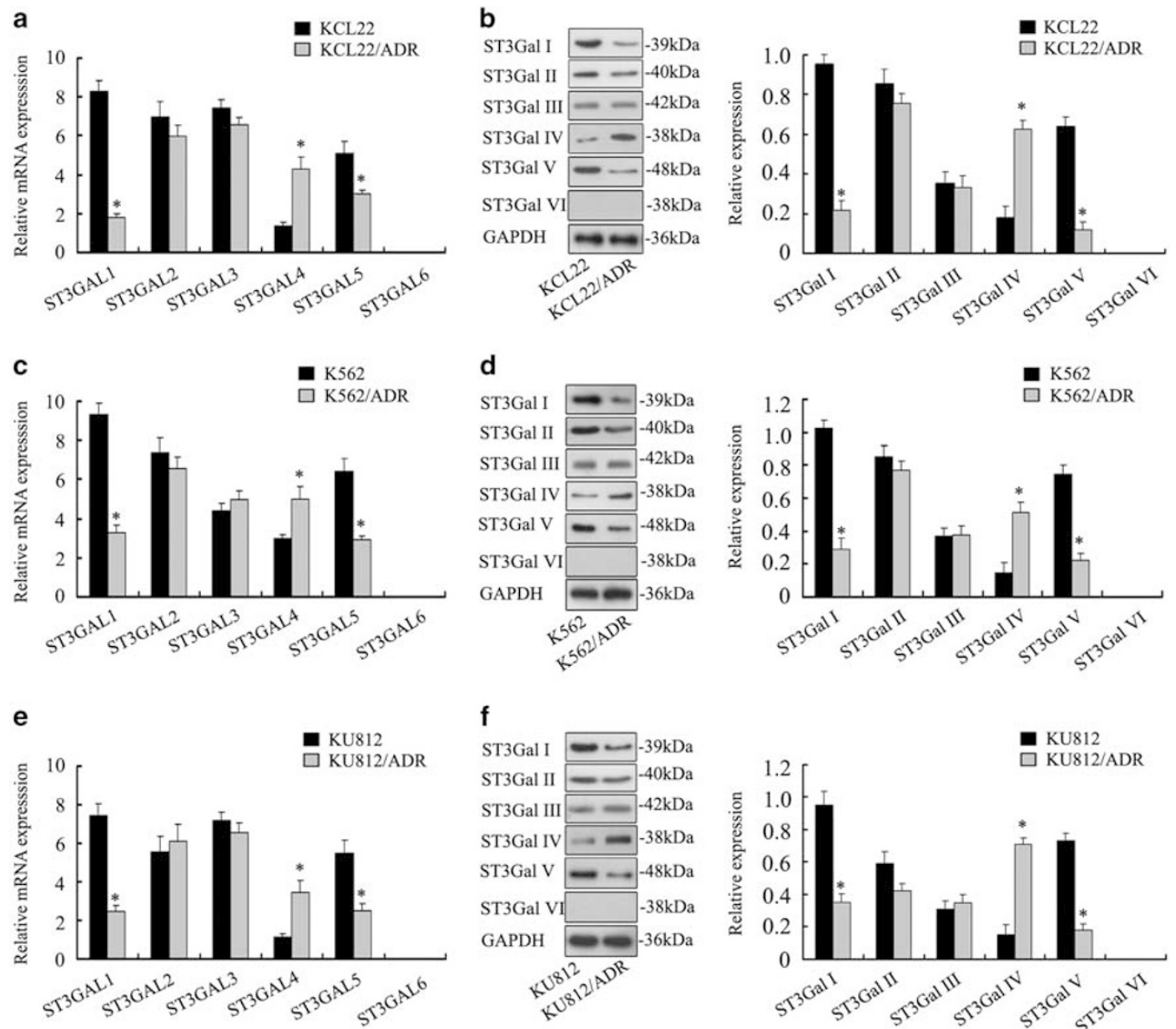



Figure 1 Differential expression of ST3GAL family in three pairs of CML cell lines. (a, $\mathbf{c}$, e) The mRNA levels of ST3GAL family were analyzed by real-time PCR. Three drug-sensitive parental cells expressed higher levels of ST3GAL1 and ST3GAL5 mRNA than their MDR cell types $\left({ }^{*} P<0.05\right)$. Three MDR cells expressed higher levels of ST3GAL4 mRNA than their parental cell types $(* P<0.05)$. No significant change of ST3GAL2 and ST3GAL3 was observed. ST3GAL6 was not detectable in MDR cells and their parental cells. (b, d, f) Western blot analysis of ST3GAL family at protein levels. GAPDH served as a control. Three MDR cells expressed higher levels of ST3Gal IV protein, although their parental cell types expressed higher levels of ST3Gal I and ST3Gal V protein $\left({ }^{*} P<0.05\right)$, expressions of the rest enzymes of ST3Gal family were also consistent with mRNA levels. Data are the mean \pm s.d. of triplicate determinants.

PCR and western blotting in order to search for abnormally expressed genes and proteins of ST3GAL family in three ADR cell lines. As shown in Figure 1, no statistically significant differences were found in the expression of ST3GAL2 and ST3GAL3 between parent cell lines KCL22, K562, KU812, and their MDR cells KCL22/ADR, K562/ADR, and KU812/ADR. Remarkable increase of ST3GAL1 and ST3GAL5 on both the gene and protein levels was observed in three drug-susceptible parental cells compared with those of three drug-resistant cell lines $\left({ }^{*} P<0.05\right)$. In addition, three drug-resistant cell lines showed higher expressional levels of ST3GAL4 $\left({ }^{\star} P<0.05\right)$. Although ST3GAL6 gene was absent in three pairs of CML cell lines.

Expression of MDR-related marker, ST3GAL gene family presenting in BMMC of CML patients was summarized in
Table 1. The group of CML without MDR showed significantly up-regulated ST3GAL1 and ST3GAL5 expression compared to $\mathrm{CML} / \mathrm{MDR}$ group $\left({ }^{\star} P<0.05\right)$. BMMC of CML/MDR patients showed higher expressional levels of ST3GAL4 $\left({ }^{*} P<0.05\right)$. Expression of ST3GAL2 and ST3GAL3 showed no difference between the two groups, although ST3GAL6 was detected slightly. These observations indicated that differential expression of ST3GAL family might associate to MDR of CML.

\section{Altered Expression of ST3GAL1 Gene Influences Chemosensitivity of CML Cells In vitro and In vivo} Because of the significant increase of ST3GAL1 expression in KCL22 cells (Figure 1a), we silenced ST3GAL1 with shRNA, in order to elucidate the direct effect on the chemosensitivity 
Table 1 Expressional profiles of ST gene family in the BMMC from CML and CML/MDR patients

\begin{tabular}{lccc}
\hline Gene & \multicolumn{2}{c}{ Relative mRNA expression $\left(\times 10^{3}\right.$, BMMC $)$} & $P$ value \\
\cline { 2 - 3 } & CML $(N=19)$ & CML/MDR $(N=29)$ & \\
\hline ST3GAL1 & $32.348 \pm 4.511$ & $20.302 \pm 3.604$ & $0.001^{*}$ \\
ST3GAL2 & $36.473 \pm 1.058$ & $36.801 \pm 1.142$ & 0.257 \\
ST3GAL3 & $13.562 \pm 1.239$ & $13.041 \pm 1.548$ & 0.170 \\
ST3GAL4 & $14.948 \pm 3.766$ & $22.105 \pm 3.341$ & $0.001^{*}$ \\
ST3GAL5 & $30.408 \pm 5.942$ & $18.115 \pm 4.158$ & $0.001^{*}$ \\
ST3GAL6 & $0.003 \pm 0.002$ & $0.004 \pm 0.002$ & 0.056 \\
\hline
\end{tabular}

${ }^{*} P<0.05$ vs $\mathrm{CML}$ patients.

of KCL22 cells. As shown in Figure 2a and b, the expression level of ST3GAL1 was significantly decreased in ST3GAL1 shRNA transfectants compared to control transfectants $\left({ }^{*} P<0.05\right), \alpha-2,3$ sialylation level detected by FITC-MAL lectin on the cell surface, was also reduced in KCL22ST3GAL1 shRNA1 cells (Figure 2c). However, there were no change of $\alpha-1,6$ fucosylation level detected by FITC-LCA lectin on the cell surface of KCL22-control shRNA and KCL22-ST3GAL1 shRNA1 cells (Figure 2c).

After ST3GAL1 shRNA transfection, the inhibition activity to the growth of KCL22 cells was evaluated with MTT assay on adriamycin, vincristine, and paclitaxel. The $\mathrm{IC}_{50}$ values (drug concentration that inhibits cell growth by 50\%) were significantly increased in KCL22-ST3GAL1 shRNA cells group compared to the control, suggesting that KCL22 cells developed MDR after treated with ST3GAL1 shRNA (Figure $2 \mathrm{~d},{ }^{\star} P<0.05$ ).

Nude mice bearing KCL22, KCL22-control shRNA, and KCL22-ST3GAL1 shRNA1 xenografts were used to determine the treatment efficacy of adriamycin by measuring tumor volumes. Primary tumor volume was found to increase with adriamycin treatment, but overall the increase was at a significantly slower rate than the untreated group (Figure 2e). In fact, up to day 21 to day 28 , a significant increase of the mean of tumor volume was measured in KCL22-ST3GAL1 shRNAl tumor $\left(891 \pm 92 \mathrm{~mm}^{3}\right)$ compared with the control shRNA group $\left(585 \pm 62 \mathrm{~mm}^{3},{ }^{\star} P<0.05\right)$. In the adriamycin treatment KCL22-ST3GAL1 shRNA1 xenograft model also

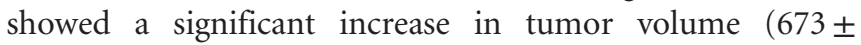
$\left.81 \mathrm{~mm}^{3},{ }^{\star} P<0.05\right)$ when compared with the control groups $\left(310 \pm 48 \mathrm{~mm}^{3},{ }^{\star} P<0.05\right)$. Furthermore, a significant reduction of mean tumor volume of adriamycin treatment KCL22-ST3GAL1 shRNA1 xenograft tumor was observed, as compared with KCL22-ST3GAL1 shRNA1 groups in the absence of adriamycin, and the effect of concomitant application of adriamycin.

Transfection of KCL22/ADR cells with ST3GAL1 cDNA resulted in an increase of ST3GAL1 expression compared to mock transfectants (Figure 3a and b). Fluorescence intensity on FITC-MAL revealed more $\alpha-2,3$ sialylation in KCL22/ ADR/ST3GAL1 cells than that in non-transfection cells (Figure 3c). However, $\alpha-1,6$ fucosylation level detected by FITC-LCA lectin on the cell surface, was no change in KCL22/ $\mathrm{ADR} /$ mock and KCL22/ADR/ST3GAL1 cells (Figure 3c). The chemoresistance of KCL22/ADR cells was decreased after the transfection of the ST3GAL1 expression vector in vitro (Figure 3d). The in vivo antitumor activity of adriamycin was also assessed using nude mice bearing KCL22/ADR, KCL22/ ADR/mock or KCL22/ADR/ST3GAL1 tumors (Figure 3e). A significant decrease of the mean of tumor volume was measured in KCL22/ADR/ST3GAL1 tumor compared with the KCL22/ADR/mock group, and in KCL22/ADR/ST3GAL1 $+\mathrm{ADR}$ tumor compared with the KCL22/ADR/mock+ADR group. Furthermore, a significant reduction of mean tumor volume of KCL22/ADR/ST3GAL1+ADR xenograft tumor was observed, as compared with KCL22/ADR/ST3GAL1 groups, and the effect of concomitant application of adriamycin. The above results indicated that ST3GAL1 mediated MDR phenotype of CML cells.

\section{MiR-4701-5p as a Negative Regulator of ST3GAL1}

Recent studies have linked leukemia MDR to the altered expression of miRNAs. We first examined the expression profiles of human miRNAs in KCL22 and KCL22/ADR cell lines using microarrays (Kangchen, Shanghai, China). The miR-4701-5p was significantly up-regulated in KCL22/ADR cells (KCL22/ADR: KCL22 $=10.2$-folds). In order to confirm and validate the findings obtained from our microarray analysis, we performed qRT-PCR analysis using three pairs of chemo-susceptible and chemoresistant human CML cell lines and the BMMC of CML patients. The results showed that miR-4701-5p was dramatically down-regulated in KCL22, K562, and KU812, compared with their chemoresistant cells, respectively (Figure 4a). In the BMMC of CML patients $(n=19)$, expression level of miR-4701-5p also was lower than that in chemoresistant group CML/MDR $(n=29$, Figure $4 a)$. The decreased expression of miR-4701-5p in chemosusceptible CML cell lines and CML patients compared with chemoresistant cells was concurrent with overexpression of ST3GAL1.

We further transfected KCL22 cells with miR-4701-5p mimics $(100 \mathrm{nM}), \quad$ KCL22/ADR cells with antagomir $(100 \mathrm{nM})$ (Figure $4 \mathrm{~b}$ ) and measured the changes in ST3GAL1 expression levels. KCL22 cells with miR-4701-5p mimics showed a significant attenuation of ST3GAL1 expression at both the mRNA and protein levels (Figure 4c), whereas the inhibition of miR-4701-5p in KCL22/ADR cells led to the opposite changes in ST3GAL1 expression (Figure 4d).

To determine whether miR-4701-5p directly regulated ST3GAL1 expression, the target sequence of ST3GAL1 3'-UTR (wt 3'-UTR) and ST3GAL1 mutant sequence (mt $3^{\prime}$-UTR) was cloned into a luciferase reporter vector. KCL22 cells were then transfected with wt or mt 3'-UTR vector and 




C
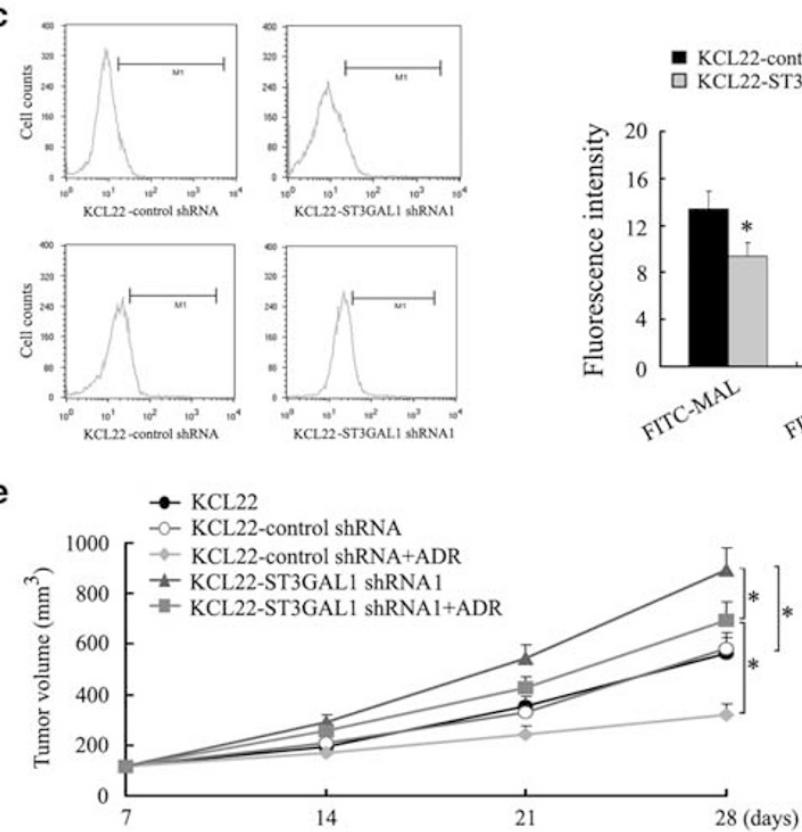

b

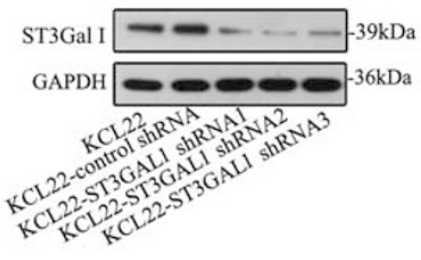

- KCL22-control shRNA CL22-ST3GAL1 ShRNA1
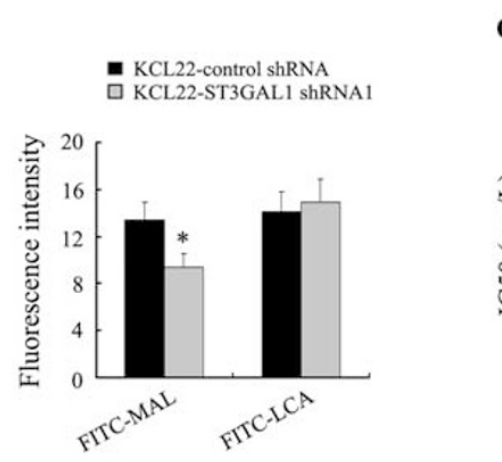

d
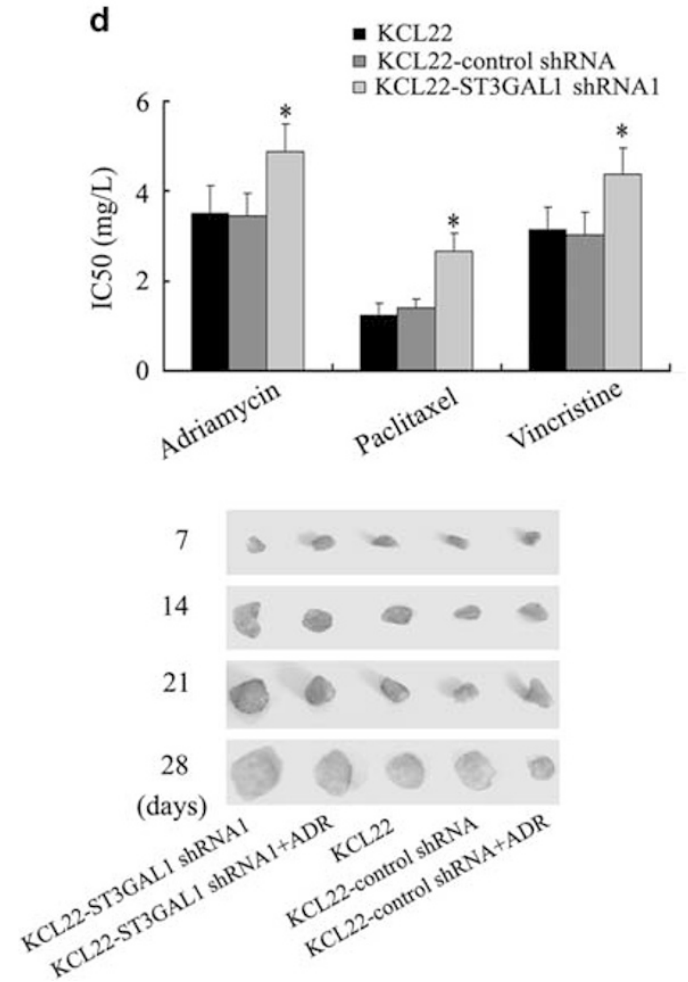

Figure 2 Silence of ST3GAL1 gene decreases the chemosensitivity of KCL22 cells both in vitro and in vivo. (a, b) Silencing of ST3GAL1 in KCL22 cells was analyzed by RNAi approach. After shRNA transfection, distinct reduction of ST3GAL1 was observed at mRNA and protein levels by real-time PCR and western blot analysis $\left({ }^{*} P<0.05\right)$. (c) Differential FITC-MAL/LCA binding profiles of KCL22-ST3GAL1 control and KCL22-ST3GAL1 shRNA1 cell lines using flow cytometry. Histograms of fluorescence intensities of cells with specific carbohydrate expression as determined. (d) Cell chemosensitivity was assessed by MTT assays. The reported values were the $\mathrm{IC}_{50}$ (mean \pm s.d.) of three independent experiments. $\mathrm{IC}_{50}$ represents the drug concentration producing $50 \%$ decrease of cell growth. ${ }^{*} P<0.05$ vs KCL22-control shRNA cells. (e) An increase of mean tumor volume in mice group with KCL22 shRNA tumors was observed, as compared with that in KCL22-control shRNA group $\left({ }^{*} P<0.05\right)$. The data are mean \pm s.d. of three independent assays $(* P<0.05)$.

miR-4701-5p mimics. Luciferase activity assays were performed. Forced miR-4701-5p expression decreased luciferase activity, and this suppression was reversed by the mutation of the target sequences in the 3'-UTRs of ST3GAL1 in KCL22 cells (Figure 4e). Taken together, these results suggested that ST3GAL1 was a direct target of miR-4701-5p in CML cells.

\section{MiR-4701-5p Modulates MDR of CML Cells by Targeting ST3GAL1 In vitro and In vivo}

To investigate whether miR-4701-5p regulated the drug sensitivity of CML cells in vitro, parental KCL22 cells were transfected with miR-4701-5p mimics, and KCL22/ADR drug-resistant cells were transfected with miR-4701-5p antagomir. The overexpression of miR-4701-5p markedly reduced chemotherapy effect to KCL22 cells with an increase in the $\mathrm{IC}_{50}$ values of adriamycin, paclitaxel, and vincristine (Figure 5a). Meanwhile, decreased $\mathrm{IC}_{50}$ values for these three chemotherapeutic agents were observed after miR-4701-5p was inhibited by antagomir in KCL22/ADR cells (Figure 5b).

To further verify whether miR-4701-5p modulated the drug sensitivity of CML cells in vivo, we transplanted KCL22 and KCL22/ADR cells that had been transfected with miR-4701-5p agomir, miR-4701-5p antagomir or negative control into nude mice. Figure $5 \mathrm{c}$ and $\mathrm{d}$ showed that a 

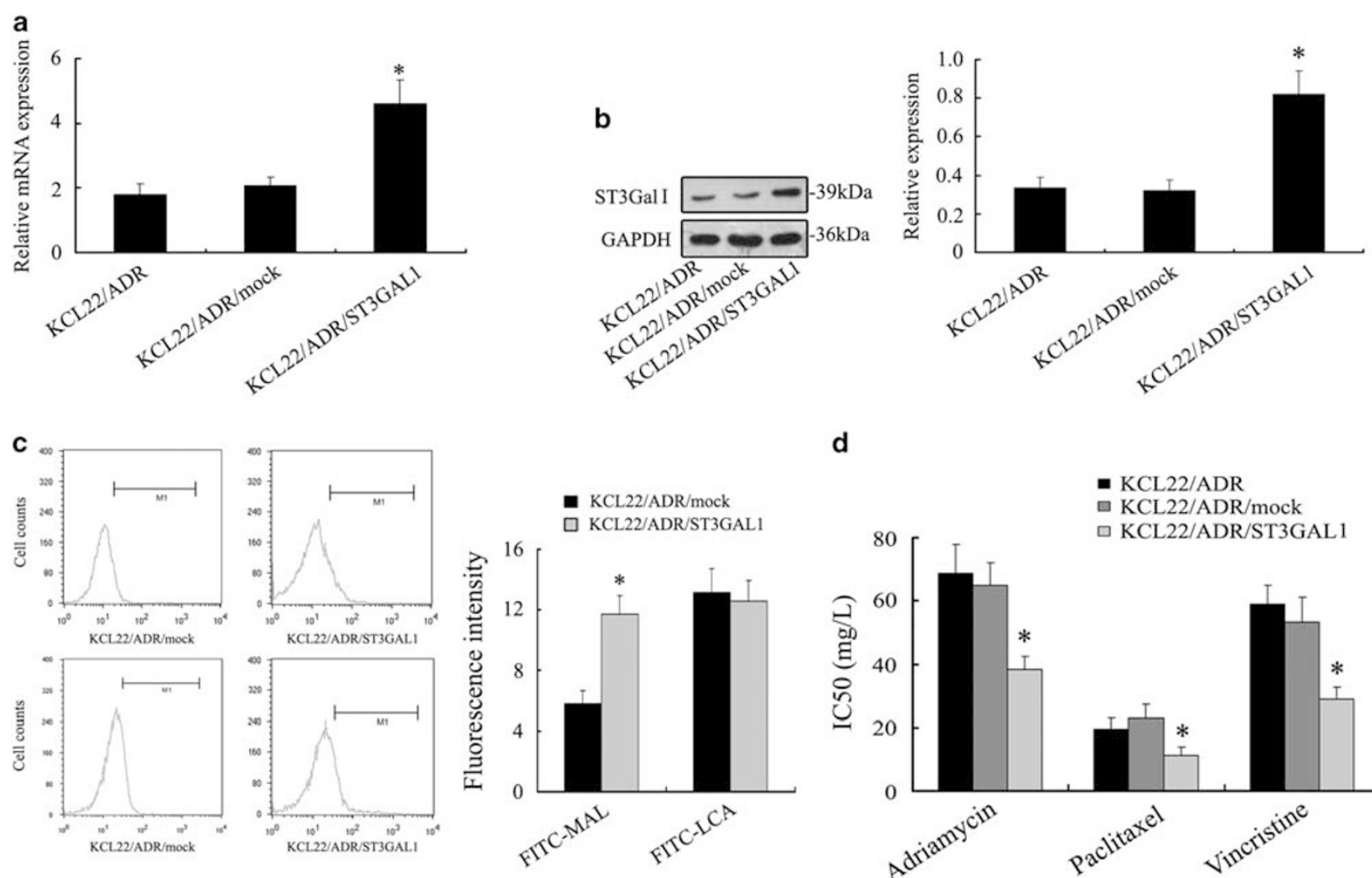

d


Figure 3 Overexpression of the ST3GAL1 gene increases the chemosensitivity of KCL22/ADR cells both in vitro and in vivo. (a, b) After full-length sequences transfection, ST3GAL1 mRNA and protein were increased notably in KCL22/ADR cells by real-time PCR and western blot analysis $(* P<0.05)$. (c) Flow cytometry analysis showed $a-2,3$ sialylation level detected by FITC-MAL on the cell surface, was also increased in KCL22/ADR/ST3GAL1 cells. The a-1, 6 fucosylation level detected by FITC-LCA on the cell surface was no change in the two cell lines. (d) Cell chemosensitivity was assessed by MTT assays. The reported values were the $I C_{50}$ (mean \pm s.d.) of three independent experiments. $I C_{50}$ represents the drug concentration producing $50 \%$ decrease of cell growth. ${ }^{*} P<0.05$ vs KCL22/ADR/mock cells. (e) A decrease of mean tumor volume in mice group with KCL22/ADR/ST3GAL1 tumor was observed, as compared with that in $\mathrm{KCL} 22 / \mathrm{ADR} /$ mock group. The data are means \pm s.d. of three independent assays $\left({ }^{*} P<0.05\right)$.

significant change of mean tumor volume of KCL22-agomir and KCL22/ADR-antagomir tumors was observed, as compared with their control groups. In the adriamycin treatment KCL22-agomir or KCL22/ADR-antagomir xenograft model, also showed a noticeable variation in tumor growth when compared with their control groups. The volumes of the
miR-4701-5p transfected tumors were remarkedly changed after ADR chemotherapy, which indicated the reversion of drug resistance by altered miR-4701-5p expression (Figure $5 c$ and d).

At the end of this experiment, the mice were killed and their tumors were isolated. RNA and protein were extracted 

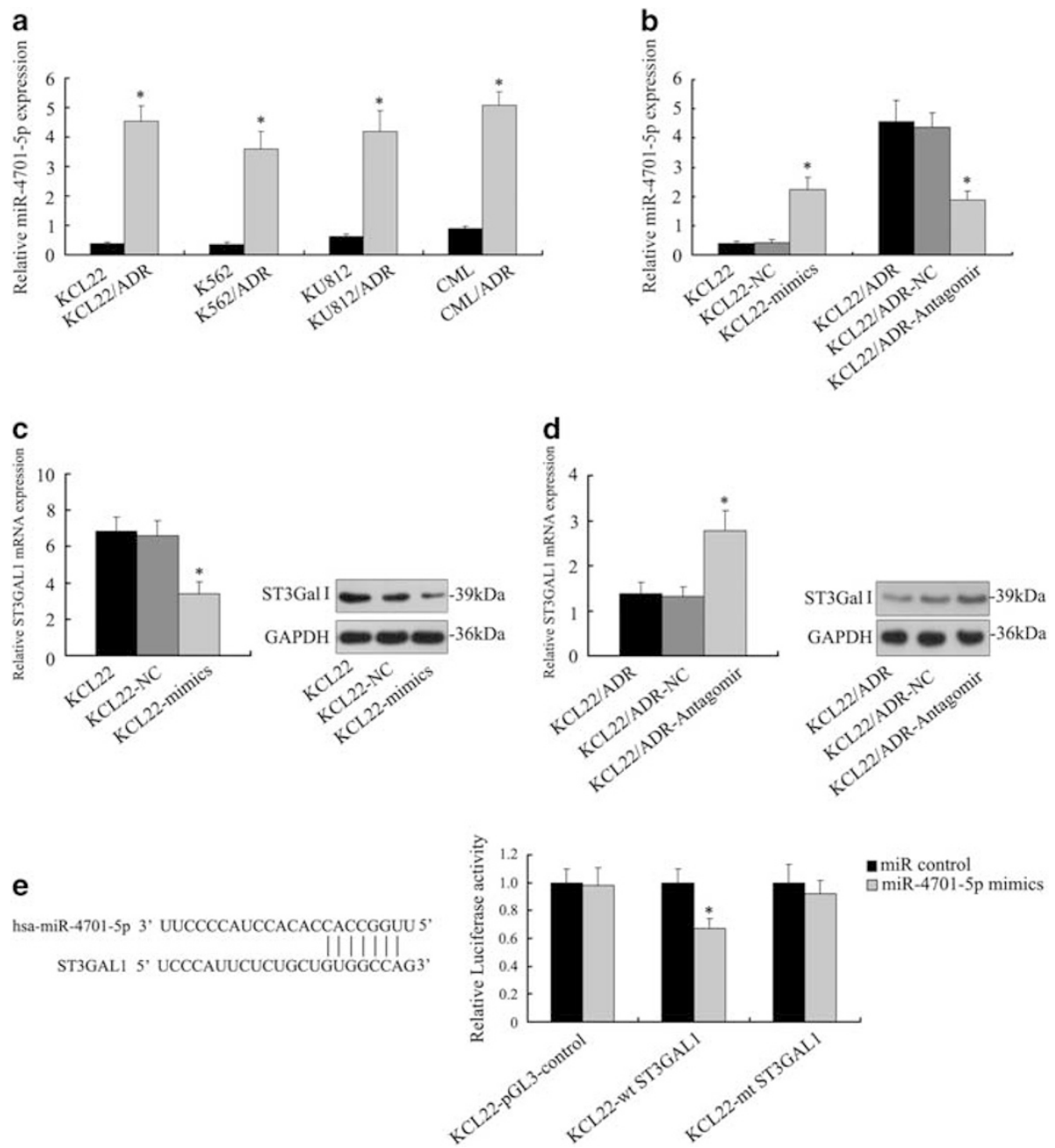

Figure 4 miR-4701-5p as a negative regulator of ST3GAL1. (a) The expression of miR-4701-5p was examined by qRT-PCR in three pairs of parental and chemoresistant human $C M L$ cell lines and CML patients $\left({ }^{*} P<0.05\right)$. (b) The expression of miR-4701-5p was studied by qRT-PCR in cells transfected with the mimics or antagomir $\left({ }^{*} P<0.05\right)$. (c, d) ST3GAL1 was detected by qRT-PCR and western blot in KCL22 and KCL22/ADR cells treated with mimics or antagomir $\left({ }^{*} P<0.05\right)$. (e) The nucleotides sequence of the target site of miR-4701 in ST3GAL1 3'-UTR (left panel); luciferase assay for the direct targeting of 3'-UTR of ST3GAL1 by miR-4701-5p (right panel). The wide-type and mutant miR-4701-5p target sequence of ST3GAL1 were fused with luciferase reporter and transfected into KCL22 cells. miR-4701-5p mimics or its miR-control was co-transfected into KCL22 cells. The mean of the results from the cells transfected with the miR-control was set at 1 . The data were mean \pm s.d. of three separate transfections $\left({ }^{*} P<0.05\right)$.

from the tumor tissues. qRT-PCR and western blotting showed that ST3GAL1 expression were regulated at both the mRNA and protein levels (Figure $5 \mathrm{~g}$ and $\mathrm{h}$ ) due to the altered expression of miR-4701-5p (Figure $5 \mathrm{e}$ and $\mathrm{f}$ ), which was consistent with the findings in vitro (Figure 5e).

\section{DISCUSSION}

Aberrant sialylation is closely associated with the malignant phenotype of cancer cells and MDR potential. STs are essential for the biosynthetic pathway of sialylated glycans. These enzymes are considered as prognostic factors and potential targets for therapeutic approaches..22,23 Remodeled mRNA expression of a panel of STs in human breast cancer, cervical cancer, ovarian cancer, and acute lymphoblastic leukemia has been documented. ${ }^{4,8,24,25}$ Here, the expression profiles of ST3GAL gene family were shown to be remodeled in three pairs of CML cell lines with real-time PCR and western blot analysis. All parent cells KCL22, K562, and KU812 were characterized by higher levels of ST3GAL1 and ST3GAL5, whereas MDR cells expressed more ST3GAL4. Other studies also suggest STs with altered mRNA expression in carcinoma tissues could be the prognostic factors and potential targets for therapeutic approaches. ${ }^{25,26}$ In this study, more than $60.4 \%$ of the CML patients were found resistant to the anticancer drugs. Six of a 2, 3-STs subtype genes, including ST3GAL1, ST3GAL2, ST3GAL3, ST3GAL4, ST3GAL5, and ST3GAL6 were used to probe the expression of ST in BMMC of CML patients. ST3GAL1 and ST3GAL5 mRNA expressed at highest level that conferred to the high levels of a 2, 3-linked NeuNAc residues on BMMC surfaces of 
a

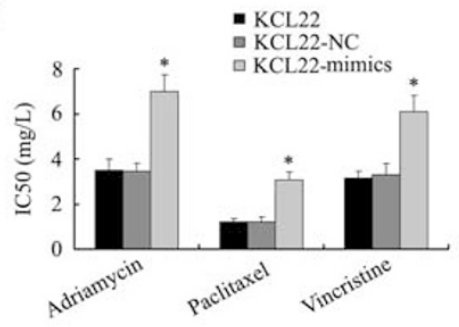

C

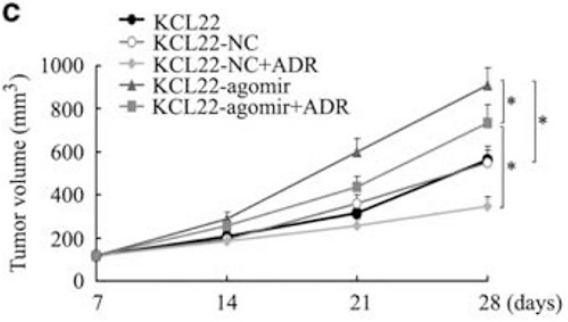

e



g

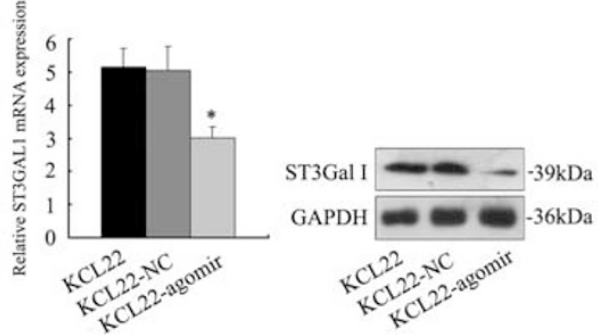

b

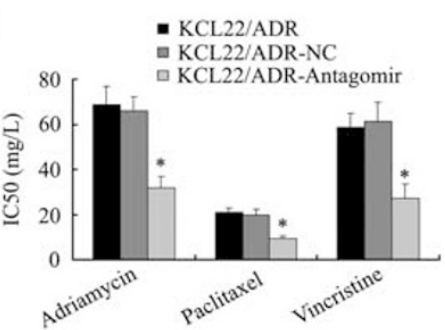

d



f

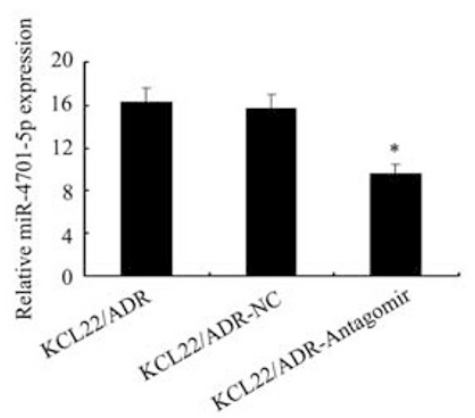

h

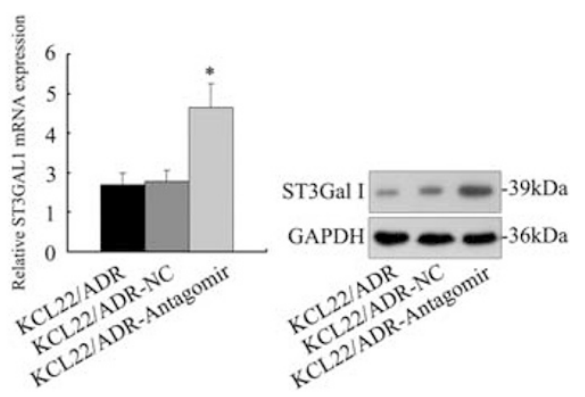

Figure 5 MiR-4701-5p modulates MDR of CML cells by targeting ST3GAL1 in vitro and in vivo. (a) Overexpression of miR-4701-5p increased IC ${ }_{50}$ values of KCL22 cells to adriamycin, paclitaxel, and vincristine $\left({ }^{*} P<0.05\right)$. (b) Knockdown of miR-4701-5p sensitized KCL22/ADR cells to adriamycin, paclitaxel, and vincristine $\left({ }^{*} P<0.05\right)$. (c, d) The tumor volumes were measured the indicated time points $(* P<0.05)$. (e, $\left.\mathbf{f}\right)$ The expression of miR-4701-5p was detected by qRT-PCR in tumors $\left({ }^{*} P<0.05\right)$. $(\mathbf{g}, \mathbf{h})$ The expression levels of ST3GAL1 were analyzed by qRT-PCR and western blots in tumors $\left({ }^{*} P<0.05\right)$. The data were mean \pm s.d. of three separate transfections $\left({ }^{*} P<0.05\right)$.

CML patients, whereas ST3GAL4 over-expressed in CML/ MDR patients. Once again the findings from the clinical samples confirmed that altered levels of these genes are associated with MDR phenotype in CML patients.

How ST3GAL family causes the MDR phenotype of CML cells? The present study targeted ST3GAL1, which showed varied expression in the three pairs of CML cell lines. ST3GAL1 product also altered remarkably in CML cell lines labeled with FITC-MAL lectin, whereas FUT8 product detected by FITC-LCA lectin didn't change on the CML cell surface targeted ST3GAL1. The altered level of ST3GAL1 led to drug-resistant phenotype changes of KCL22 and KCL22/ ADR cells both in vitro and in vivo. On the basis of the above results, it implies the utilization of ST3GAL1 as a biomarker for clinical diagnosis and prognosis of MDR of CML and as potential targets for therapeutic approaches in future.

Former studies demonstrated an involvement of miRNAs in the development of drug resistance in cancer. Altered 
expression of miRNAs, a common feature of cancer, has recently been linked to epigenetic mechanisms. ${ }^{27,28,29}$ In the present study, miR-4701-5p expression in three pairs of chemo-sensitive human CML cell lines and the PBMC of CML patients was significantly lower than that of chemoresistant cell lines and CML/ADR patients. Compared with chemoresistant CML cells, the decreased expression of miR-4701-5p in parental CML cells was concurrent with ST3GAL1 overexpression. Furthermore, we found that miR-4701-5p directly targeted sequences within the 3'-UTR of ST3GAL1, thus decreasing ST3GAL1 expression at the mRNA and protein levels.

Alternative approaches for regulating the expression of endogenous miRNA should be explored further to validate this therapeutic concept. The current study demonstrated that the ectopic expression of miR-4701-5p converted KCL22 and KCL22/ADR cells to susceptible to adriamycin, paclitaxel, and vincristine in vitro. Altered expression of miR-4701-5p significantly regulated the ADR-mediated growth of xenografts in mice. This result indicated that miR-4701-5p might serve as a MDR-specific miRNA in human CML by targeting ST3GAL1.

In conclusion, the differential expression patterns of $\alpha-2,3-$ STs and miR-4701-5p were shown in three pairs of CML cell lines and in BMMC of the CML patients with and without MDR. MiR-4701-5p was found to regulate CML cells MDR by targeting ST3GAL1. Although the modification of miR-4701-5p has been indicated as a causative factor for the MDR phenotype, there could be other potential mechanisms involved. Therefore, molecular basis underlying leukemia MDR-associated phenotype remained to be investigated further.

\section{ACKNOWLEDGMENTS}

This work was supported by grants from the National Key Basic Research and Development Program (973 program) of China (No. 2012CB822100), from the National Natural Science Foundation of China $(81271910,81472014)$, and from the Natural Science Foundation of Liaoning Province, China (2014023043).

\section{DISCLOSURE/CONFLICT OF INTEREST}

The authors declare no conflict of interest.

1. Sloma I, Jiang $X$, Eaves $A C$ et al. Insights into the stem cells of chronic myeloid leukemia. Leukemia 2010;24:1823-1833.

2. Estey EH. Cellular mechanisms of multidrug resistance of tumor cells. Biochemistry (Mosc) 2000;65:95-106.

3. Zhang Z, Zhao $Y$, Jiang $L$ et al. Glycomic alterations are associated with multidrug resistance in human leukemia. Int J Biochem Cell Biol 2012;44:1244-1253.

4. Nakana M, Saldanha R, Göbel A et al. Identification of glycan structure alterations on cell membrane proteins in desoxyepothilone $B$ resistant leukemia cells. Mol Cell Proteomics 2011;10:M111.009001.
5. Harduin-Lepers A, Vallejo-Ruiz V, Krzewinski-Recchi MA et al. The human sialyltransferase family. Biochimie 2001;83:727-737.

6. Videira PA, Correia M, Malagolini $\mathrm{N}$ et al. ST3Gal I sialyltransferase relevance in bladder cancer tissues and cell lines. BMC Cancer 2009;9:357.

7. Saito S, Aoki H, Ito A et al. Human alpha2, 3-sialyltransferase (ST3Gal II) is a stage-specific embryonic antigen-4 synthase. J Biol Chem 2003;278:26474-26479.

8. Recchi MA, Hebbar M, Hornez L et al. Multiplex reverse transcription polymerase chain reaction assessment of sialyltransferase expression in human breast cancer. Cancer Res 1998:58:4066-4070.

9. Jun L, Yuanshu W, Yanying $X$ et al. Altered mRNA expressions of sialyltransferases in human gastric cancer tissues. Med Oncol 2012;29: 84-90.

10. Mondal S, Chandra S, Mandal C. Elevated mRNA level of hST6Gal I and hST3Gal V positively correlates with the high risk of pediatric acute leukemia. Leukemia Res 2010;34:463-470.

11. Okajima T, Fukumoto S, Miyazaki $\mathrm{H}$ et al. Molecular cloning of a novel alpha2,3-sialyltransferase (ST3Gal VI) that sialylates type II lactosamine structures on glycoproteins and glycolipids. J Biol Chem 1999;274: 11479-11486.

12. Chen CZ. MicroRNAs as oncogenes and tumor suppressors. N Engl J Med 2005;353:1768-1771.

13. Ambros V. The functions of animal microRNAs. Nature 2004;431: 350-355.

14. Brennecke J, Cohen S. Towards a complete description of the microRNA complement of animal genomes. Genome Biol 2003:4:228.

15. Fabbri $M$, Croce CM. Role of microRNAs in lymphoid biology and disease. Curr Opin Hematol 2011;18:266-272.

16. Blower PE, Chung JH, Verducci JS et al. MicroRNAs modulate the chemosensitivity of tumor cells. Mol Cancer Ther 2008;7:1-9.

17. Passetti F, Ferreira CG, Costa FF. The impact of microRNAs and alternative splicing in pharmacogenomics. Pharmacogenomics $J$ 2009;9:1-13.

18. Lu F, Zhang J, Ji M et al. miR-181b increases drug sensitivity in acute myeloid leukemia via targeting HMGB1 and Mcl-1. Int J Oncol 2014;45: 383-392.

19. Xu Y, Ohms SJ, Li Z et al. Changes in the expression of miR-381 and miR-495 are inversely associated with the expression of the MDR1 gene and development of multi-drug resistance. PLoS One 2013;8: e82062.

20. Feng DD, Zhang $H$, Zhang $P$ et al. Down-regulated miR-331-5p and miR-27a are associated with chemotherapy resistance and relapse in leukaemia. J Cell Mol Med 2011;15:2164-2175.

21. Zhao $X$, Yang $L$, Hu J et al. miR-138 might reverse multidrug resistance of leukemia cells. Leuk Res 2010;34:1078-1082.

22. Zhu Y, Srivatana U, Ullah A et al. Suppression of a sialyltransferase by antisense DNA reduces invasiveness of human colon cancer cells in vitro. Biochim Biophys Acta 2001;1536:148-160.

23. Cui $H, L$ in $Y, Y u e ~ L$ et al. Differential expression of the a2,3-sialic acid residues in breast cancer is associated with metastatic potential. Oncol Rep 2011;25:1365-1371.

24. Wang $\mathrm{PH}, \mathrm{Li} \mathrm{YF}$, Juang $\mathrm{CM}$ et al. Altered mRNA expression of sialyltransferase in squamous cell carcinomas of the cervix. Gynecol Oncol 2001;83:121-127.

25. Wang $\mathrm{PH}$, Lee $\mathrm{YR}$, Juang $\mathrm{CM}$ et al. Altered mRNA expression of sialyltransferases in ovarian cancers. Gynecol Oncol 2005;99:631-639.

26. López-Morales D, Velázquez-Márquez N, Valenzuela O et al. Enhanced sialyltransferases transcription in cervical intraepithelial neoplasia. Invest Clin 2009;50:45-53.

27. Esquela-Kerscher A, Slack FJ. Oncomirs-microRNAs with a role in cancer. Nat Rev Cancer 2006:6:259-269.

28. Lujambio A. CpG island hypermethylation of tumor suppressor microRNAs in human cancer. Cell Cycle 2007;6:1454-1458.

29. Croce CM. Causes and consequences of microRNA dysregulation in cancer. Nat Rev Genet 2009;10:704-714. 